Nervenarzt 2021 · 92:762-772

https://doi.org/10.1007/s00115-021-01138-5

Angenommen: 29. April 2021

Online publiziert: 7. Juni 2021

(c) Der/die Autor(en) 2021

\section{Hintergrund}

Die mechanische Thrombektomie (MT) wurde im Jahr 2015 nach der Veröffentlichung von fünf unabhängigen randomisiert-kontrollierten Studien (RCTs) das Standardverfahren für die Behandlung eines akuten ischämischen Schlaganfalls (AIS) aufgrund eines proximalen Arterienverschlusses („large vessel occlusion“, LVO) der vorderen Zirkulation im Zeitfenster von 0-6h. Der klinische Nutzen war mit einer "number needed to treat“ von ca. 2,5 für die Reduktion neurologischer Langzeitfolgen überwältigend [1]. Allerdings führten die strengen Einschlusskriterien der oben genannten Studien dazu, dass nur ca. einer von 17 Schlaganfallpatienten sich für diese Behandlung qualifizierte [2]. Aktuelle Studien weisen darauf hin, dass der Anteil an Schlaganfallpatienten, die von der MT profitieren könnten, mit ca. $22 \%$ deutlich höher ist $[3,4]$.

In diesem Review geben wir daher einen Überblick über die aktuelle Literatur insbesondere im Hinblick auf $\mathrm{Pa}$ tientenkollektive, die in den bisherigen RCTs unterrepräsentiert waren. Zusätzlich beleuchten wir aktuelle Neuentwicklungen in der interventionellen Behandlung von AIS-Patienten. Abschließend fassen wir die Empfehlungen der aktuel-

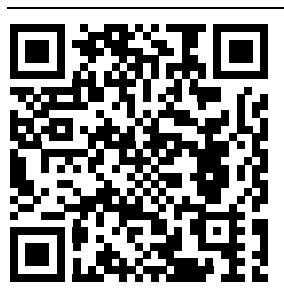

QR-Code scannen \& Beitrag online lesen

Marios-Nikos Psychogios ${ }^{1}$ Alex Brehm ${ }^{1}$ Peter Sporns ${ }^{1}$ Leo H. Bonati ${ }^{2}$

${ }^{\prime}$ Abteilung für interventionelle und diagnostische Neuroradiologie, Klinik für Radiologie und Nuklearmedizin, Universitätsspital Basel, Basel, Schweiz

${ }^{2}$ Hirnschlagzentrum, Klinik für Neurologie, Universitätsspital Basel, Basel, Schweiz

\title{
Grenzbereiche der Thrombektomie
}

len Guidelines der amerikanischen Herzund Schlaganfallorganisation (American Heart Association/American Stroke Association, AHA/ASA) und der europäischen Schlaganfallorganisation (ESO) im Hinblick auf die oben angesprochenen Patientenkollektive zusammen $[5,6]$. Ergänzend geben wir auch eine eigene Empfehlung auf Basis der hier besprochenen Literatur (• Tab. 1).

\section{Mögliche Indikations- erweiterungen für MT}

\section{Zeitdauer zwischen Beginn der Symptome und Präsentation im Krankenhaus}

In den fünf Studien, die $2015 \mathrm{zu}$ einem Paradigmenwechsel in der Therapie des AIS aufgrund eines LVO der vorderen Zirkulation führten, präsentierte sich nur ein Bruchteil der Patienten (5\% von 1287 Patienten) in einem Zeitfenster von mehr als $6 \mathrm{~h}$ [1]. Allerdings zeigte sich bereits in der ersten Metaanalyse (HERMES) der oben genannten Studien ein statistisch signifikanter positiver Effekt auf das klinische Ergebnis (Odds Ratio [OR] 1,76; $95 \%$-Confidence-Intervall [CI $1,05-2,97)$ auch bei Patienten, die sich mindestens $5 \mathrm{~h}$ nach den ersten Symptomen präsentierten [1].

Im Jahr 2018 zeigten die DAWN- und DEFUSE-3-Studien, dass bei ausgewählten Patienten die MT auch im Zeitfenster von $6-24 \mathrm{~h} \mathrm{zu}$ einem deutlich besseren langfristigen neurologischen Ergebnis (definiert als „modified Rankin Scale“ [mRS] nach 90 Tagen) führt als die bisher standardmäßig genutzte medikamentöse Therapie [7, 8]. Das Besondere an diesen
Studien war, dass sie als primäres Einschlusskriterium ein radiologisches Mismatch entweder zwischen dem Infarktkern und der noch „rettbaren“ Penumbra (DEFUSE-3; [8]) oder zwischen dem Infarktkern und der klinischen Präsentation (DAWN; [7]) voraussetzten. Die sehr strengen Einschlusskriterien beider Studien führten dazu, dass aktuell nur ca. $30 \%$ der LVO-Patienten, die sich im Zeitfenster von 6-24 $\mathrm{h}$ befinden, nach der aktuellen AHA/ASA-Guideline eine klare Indikation für die MT aufweisen [9]. Nichtrandomisierte Studien legen allerdings nahe, dass der Nutzen einer MT auch bei Patienten vorhanden ist, die nach den DAWN- und DEFUSE-3-Kriterien keine Kandidaten für die MT wären $[9,10]$. Weiterhin konnte auch gezeigt werden, dass eine Patientenselektion auf Basis der Kollateralen zu ähnlich guten Behandlungsergebnissen führt wie die perfusionsbasierte Selektion, die in den DAWN- und der DEFUSE-3-Studien genutzt wurde [11].

\section{) Bei ausreichend "rettbarer" Penumbra sollte die MT auch im späten Zeitfenster erwogen werden}

Die aktuell laufenden Studien RESILIENT Extend (NCT02216643) und MR CLEAN LATE (ISRCTN19922220) untersuchen die Fragestellung, ob ein vereinfachtes bildgebendes Verfahren ohne CT(Computertomographie)-Perfusion oder Magnetresonanztomographie (MRT) für die Patientenselektion im Zeitfenster von mehr als $6 \mathrm{~h}$ genutzt 
Tab. 1 Übersicht über erweiterte Behandlungsindikationen für die mechanische Thrombektomia ${ }^{a}$

Zeit von Bei Patienten mit einem Verschluss der Symptom- vorderen Zirkulation, die sich zwischen 6 beginn/ und $24 \mathrm{~h}$, nachdem sie zuletzt gesund zuletzt gesehen wurden, präsentieren, wird eine gesund ge- MT empfohlen, sofern die DAWN- oder sehen zum DEFUSE-3-Kriterien erfüllt sind Behandlungsbeginn

\section{Grad der Evidenz: moderat $\oplus \oplus \oplus$}

Stärke der Empfehlung: stark $\uparrow$

Expertenmeinung: Im Zeitfenster von 6-12 h können Patienten, die die ESCAPE-Kriterien (moderate bis gute Kollateralen und $\mathrm{ASPECTS} \geq 6$ ) erfüllen durch MT behandelt werden

Leichtere AIS-Patienten mit milder Symptomatik Schlagan- (NIHSS 0-5) und großem Gefäßverschluss, fälle (NIHSS die sich bis zu $24 \mathrm{~h}$, nach dem sie zuletzt <6) gesund gesehen wurden, vorstellen, sollten in RCTs eingeschlossen werden

Grad der Evidenz: sehr niedrig $\oplus$

Stärke der Empfehlung: neutral -

Größe des AIS-Patienten mit einem Verschluss der Infarktes vorderen Zirkulation mit großem Infarkt(ASPECTS) kern (ASPECTS $<6$ oder Core-Volumen $>70 / 100 \mathrm{ml}$ ) sollten in RCTs zur Evaluierung des Nutzens der MT gegenüber medizinischem Management eingeschlossen werden

\section{Grad der Evidenz: sehr niedrig $\oplus$} Stärke der Empfehlung: neutral -

MT in Kom- AIS-Patienten mit einem LVO sollten eine bination kombinierte Therapie aus MT und i.v. TPA mit i.v. IPA erhalten, sofern keine Kontraindikation besteht. Beide Behandlungen sollten so schnell wie möglich erfolgen und sich nicht gegenseitig verzögern

Grad der Evidenz: sehr niedrig $\oplus$

Stärke der Empfehlung: stark $\uparrow$

Expertenmeinung: Sofern ein LVO nachgewiesen wird vor i.v. tPA-Gabe, sollte Tenecteplase bevorzugt werden

Distale Expertenmeinung: Bei M2-Verschlüssen Verschlüsse besteht Konsensus unter den Experten, dass eine MT angemessen ist. Keine Aussage zu anderen distalen Verschlüssen

Verschlüsse Expertenmeinung: Bei Verschlüssen der der hin- A. basilaris besteht Konsensus unter den teren Zir- Experten, dass eine MT angemessen ist. kulation Keine spezifische Aussage zu den Aa. posterior und vertebralis
Bei Patienten mit AIS aufgrund eines Verschlusses der vorderen Zirkulation, die sich zwischen 6 und $16 \mathrm{~h}$ nach Symptombeginn, nach dem sie zuletzt gesund gesehen wurden, vorstellen und die DAWN- oder DEFUSE-3-Kriterien erfüllen wird eine Thrombektomie empfohlen

\section{Grad der Evidenz: A}

Stärke der Empfehlung: stark $\uparrow$

Bis zu $24 \mathrm{~h}$ ist eine mechanische Thrombektomie bei Patienten, die die DAWN Kriterien erfüllen angemessen

Grad der Evidenz: B-R

Stärke der Empfehlung: moderat $\uparrow$

Auch wenn der Nutzen unklar ist, kann bei Patienten mit einem proximalen Verschluss der vorderen Zirkulation eine MT auch bei einem NIHSS $<6$ in einem Zeitfenster von $6 \mathrm{~h}$ durchgeführt werden

\section{Grad der Evidenz B-R}

Stärke der Empfehlung: schwach $\uparrow$ ?

Auch wenn der Nutzen unklar ist, kann bei Patienten mit einem proximalen Verschluss der vorderen Zirkulation eine MT auch bei eine ASPECTS $<6$ in einem Zeitfenster von $6 \mathrm{~h}$ durchgeführt werden

\section{Grad der Evidenz B-R}

Stärke der Empfehlung: schwach $\uparrow$ ?

AIS-Patienten bei denen i.v. tPA infrage

kommt, sollten sie erhalten, auch wenn eine

MT durchgeführt werden soll

\section{Grad der Evidenz A}

Stärke der Empfehlung: stark $\uparrow$

Es könnte vernünftig sein, bei MT-Patienten Tenecteplase statt Alteplase zu verwenden

\section{Grad der Evidenz B- $R$}

Stärke der Empfehlung: schwach $\uparrow$ ?

Bei gut ausgewählten AIS-Patienten auf Basis eines M2- oder M3-Verschlusses könnte eine MT innerhalb der ersten $6 \mathrm{~h}$ nach Symptombeginn von Vorteil sein

\section{Grad der Evidenz: $B-R$}

Stärke der Empfehlung: schwach $\uparrow$ ?

Bei gut ausgewählten AIS-Patienten auf Basis Wir empfehlen bei Verschlüssen der hinteeines Verschlusses der A. basilars, A. vertebralis ren Zirkulation die Durchführung der MT oder A. posterior könnte eine MT innerhalb der ersten $6 \mathrm{~h}$ nach Symptombeginn von Vorteil sein

Grad der Evidenz: C-LD

Stärke der Empfehlung: schwach $\uparrow$ ?
Auch in einem Zeitfenster von $>24 \mathrm{~h}$ sollte eine Thrombektomie erwogen werden, sofern in der (Perfusions-)Bildgebung noch eine relevante Penumbra nachgewiesen werden kann

Bis zum Vorliegen besserer Evidenz sollte Abwägung der potenziellen Risiken und Chancen erfolgen

Basierend auf der bisherigen Evidenz empfehlen wir die Erwägung einer MT bei Patienten mit einem ASPECTS von 4-6 und bei ausgewählten Patienten (frühes Zeitfenster, Alter $<70$ Jahre, vorher selbstständig zu Hause lebend) auch mit einem ASPECTS von $0-3$

Wir empfehlen weiterhin die Gabe von i.v. tPA, auch wenn eine MT durchgeführt werden soll. Die Gabe sollte die MT auf keinen Fall verzögern

Tenecteplase sollte als Alternative zur derzeit genutzten Alteplase erwogen werden, insbesondere bei Patienten mit nachgewiesenem LVO

Wir empfehlen bei Patienten mit distalen Verschlüssen, die sicher per MT erreichbar sind, eine MT durchzuführen, sofern ein klinischer Vorteil zu erwarten ist. Dies gilt unabhängig vom Zeitfenster (1) eine MT nur im Einzelfall nach genauer 
werden kann. Sollten diese Studien positiv sein, würde dies zu einer deutlichen Erweiterung der Indikation für die MT führen. Weiterhin legen neuere Forschungsergebnisse nah, dass Patienten mit radiologisch nachweisbarer Penumbra von der MT selbst in einem Zeitfenster von bis zu 10 Tagen profitieren können [12]. Die MT sollte auch im sehr späten Zeitfenster bei allen LVOPatienten erwogen werden, sofern sich ausreichende „rettbare“ Penumbra in der Perfusionsbildgebung nachweisen lässt.

\section{Ausgedehnte Infarktfrühzeichen}

Bei fast allen RCTs waren ausgedehnte Infarktfrühzeichen, definiert als ein Alberta Stroke Program CT Score (ASPECTS) $<6$, ein Ausschlusskriterium. Dennoch wurden in einigen Studien auch Patienten mit niedrigeren ASPECTS eingeschlossen. In einer 2018 veröffentlichen Metaanalyse von 7 RCTs $(n=1764)$ zeigte sich auch bei Patienten mit einem ASPECTS von 5-7 (OR 1,58; 95\%-CI 1,19-2,11) sowie mit einem ASPECTS von 0-4 (OR 2,15 ; $95 \%$-CI 1,06-4,37) ein positiver Effekt auf das neurologische Outcome. Allerdings sind insbesondere die Ergebnisse für einen ASPECTS von 0-4 aufgrund der niedrigen Stichprobe $(n=126)$ mit erheblicher Unsicherheit behaftet [13].

Damit übereinstimmend zeigte eine Metaanalyse, die auch nichtrandomisierte Studien einschloss, bei Patienten mit einem ASPECTS von 0-6 einen deutlichen Effekt der MT. So erreichten in der MTGruppe $30 \%$ ein gutes neurologisches Ergebnis (definiert als mRS $\leq 2$ nach 90 Tagen), während dies in der medikamentös behandelten Gruppe nur bei 3,2\% der Fall war. Subgruppenanalysen zeigten aber, dass mit steigender Infarktgröße die Quote der Patienten mit gutem neurologischem Ergebnis auch in der MTGruppe deutlich abnimmt. Erreichten bei den Patienten mit einem ASPECTS von 4 noch $22,1 \%$ ein gutes neurologisches Ergebnis, so waren es in der Gruppe mit einem ASPECTSvon 0-3 nur noch 13,9\% [14].

Zusammenfassend reicht die bisherige Evidenz nicht für eine abschließende Bewertung der Sicherheit und Effizienz der MT bei dieser Patien-

Nervenarzt 2021 -92:762-772 https://doi.org/10.1007/s00115-021-01138-5

(c) Der/die Autor(en) 2021

\section{M.-N. Psychogios · A. Brehm · P. Sporns · L. H. Bonati}

\section{Grenzbereiche der Thrombektomie}

\section{Zusammenfassung}

Die mechanische Thrombektomie (MT) hat sich als Standardverfahren für die Behandlung akuter ischämischer Schlaganfälle aufgrund eines Verschlusses einer großen, proximalen Hirnarterie der vorderen Zirkulation etabliert. Dennoch sind nach aktuellen Guidelines noch große Patientenkollektive von dieser hocheffektiven Behandlungsmethode ausgeschlossen. Diese Arbeit gibt daher einen Überblick über mögliche Erweiterungen der Behandlungsindikationen für die MT, wie z. B. Patienten im erweiterten Zeitfenster, mit distalen Verschlüssen, mit großem Infarktkern oder auch für sehr alte ( $>90$ Jahre) und junge (0-17 Jahre) Patienten. Zusätzlich besprechen wir neue Entwicklungen in der interventionellen Behandlung von Schlaganfällen, wie z. B. neue Triage-Konzepte oder die Fragestellung, ob die zusätzliche intravenöse Thrombolyse bei MT-Patienten notwendig ist. Abschließend geben wir für die besprochenen Behandlungsindikationen unsere Einschätzungen basierend auf der aktuellen Literatur und unserer klinischen Erfahrung.

Schlüsselwörter

Ischämischer Schlaganfall · Mechanische Thrombektomie · Endovaskuläre Therapie · Gefäßverschluss · Behandlungsindikationen

\section{Border areas of thrombectomy}

\section{Abstract}

Mechanical thrombectomy (MT) has become the standard procedure in the treatment of patients with acute ischemic stroke (AIS) due to occlusion of a large proximal cerebral artery of the anterior circulation. Nevertheless, according to the current guidelines large patient collectives are still excluded from this highly effective treatment method. Therefore, this article gives an overview of possible extensions of the indications for treatment with MT. For example, patients in the extended time window with distal occlusions, with large infarct cores and also for very old $(90+$ years) or young ( $0-17$ years) patients. Furthermore, we discuss recent developments in the interventional treatment of stroke, such as new triage concepts or the question whether an additional intravenous thrombolysis is necessary in patients with MT. We conclude with our own estimations for the discussed indications for treatment based on our clinical experience and the current literature.

\section{Keywords}

Ischemic stroke - Mechanical thrombectomy . Endovascular treatment - Vascular occlusion . Treatment indications tengruppe aus. Dies wurde auch in einem Konsensus-Statement der ESO, der European Society for Minimally Invasive Neurological Therapy (ESMINT) und der European Society of Neuroradiology zum Ausdruck gebracht [15]. Dies führte zur Initiierung mehrerer randomisiert-kontrollierter Studien, wie den europäischen TENSIONund LASTE-Studien (ClinicalTrials.gov NCT03094715 \& NCT03811769), der amerikanischen TESLA-Studie (ClinicalTrials.gov NCT03805308) sowie der japanischen RESCUE-Japan-LIMIT (ClinicalTrials.gov; NCT03702413)-Studie.

Basierend auf der bisherigen Evidenz empfehlen wir die Erwägung einer MT bei Patienten mit einem ASPECTS von 4-6 und bei ausgewählten Patienten (frühes Zeitfenster, Alter $<70$ Jahre, vorher selbstständig zu Hause lebend) auch mit einem ASPECTS von 0-3.

\section{Distale Verschlüsse}

In den bisherigen RCTs zur Zulassung der MT (bis auf MR CLEAN und EXTEND IA) war ein wesentliches Einschlusskriterium ein LVO der vorderen Zirkulation [1, 13]. Unter einem LVO wird meistens ein Verschluss des M1Segments der A. cerebri media sowie ein Verschluss der A. carotis interna verstanden [1]. Aufgrund der unklaren Datenlage ist ein weiter distal gelegener 


\begin{tabular}{|c|c|c|c|}
\hline Indikation & ESO/ESMINT-Guidelines [6] & AHA/ASA-Guidelines [5] & $\begin{array}{l}\text { Aktuelle Forschungsergebnisse und } \\
\text { Empfehlung der Autoren }\end{array}$ \\
\hline \multirow[t]{4}{*}{$\begin{array}{l}\text { Tandem- } \\
\text { Verschlüsse }\end{array}$} & $\begin{array}{l}\text { Der intrakranielle LVO sollte behandelt } \\
\text { werden, hinsichtlich der Behandlungsmo- } \\
\text { dalität für die extrakranielle Läsion kann } \\
\text { keine Empfehlung gegeben werden. Diese } \\
\text { Patienten sollten in RCTs eingeschlossen } \\
\text { werden }\end{array}$ & $\begin{array}{l}\text { Die Behandlung des/der extrakraniellen Ver- } \\
\text { schlusses/Stenose während der primären } \\
\text { Thrombektomie könnte vernünftig sein }\end{array}$ & \multirow[t]{4}{*}{$\begin{array}{l}\text { Wir empfehlen die Durchführung der MT } \\
\text { auch bei Tandem-Verschlüssen sowie, wenn } \\
\text { sicher möglich, das parallele primäre Sten- } \\
\text { ting der extrakraniellen Läsion }\end{array}$} \\
\hline & Grad der Evidenz: sehr niedrig $\oplus$ & Grad der Evidenz $B-R$ & \\
\hline & Stärke der Empfehlung: neutral - & \multirow[t]{2}{*}{ Stärke der Empfehlung: schwach $\uparrow ?$} & \\
\hline & $\begin{array}{l}\text { Expertenmeinung: } 9 / 11 \text { Experten sofern } \\
\text { kein Einschluss in RCT möglich, sollte bei } \\
\text { hochgradiger Stenose am ehesten ein } \\
\text { Stenting erwogen werden }\end{array}$ & & \\
\hline $\begin{array}{l}\text { Schlaganfall } \\
\text { bei Kindern } \\
\text { und Ju- } \\
\text { gendlichen } \\
\text { (0-17 Jah- } \\
\text { re) }\end{array}$ & Keine spezifische Empfehlung & $\begin{array}{l}\text { In Abwesenheit besserer Studiendaten bleibt } \\
\text { eine Behandlung von Kindern mit LVO durch } \\
\text { MT umstritten [84] }\end{array}$ & $\begin{array}{l}\text { Wir empfehlen die Durchführung von MT } \\
\text { auch bei Kindern und Jugendlichen, sofern } \\
\text { sie die Kriterien der bisherigen RCTs zur MT } \\
\text { bei Erwachsenen erfüllen } \\
\text { Sofern als Ursache eine Arteriopathie ver- } \\
\text { mutet wird, sollte mit besonderer Vorsicht } \\
\text { agiert werden }\end{array}$ \\
\hline \multirow{6}{*}{$\begin{array}{l}\text { Schlaganfall } \\
\text { bei sehr } \\
\text { alten } \\
\text { Patienten } \\
\text { (>90 Jahre) }\end{array}$} & $\begin{array}{l}\text { Ein oberes Alterslimit für die MT ist nicht } \\
\text { gerechtfertigt bei AIS-Patienten, die sich } \\
\text { innerhalb von } 6 \mathrm{~h} \text { vorstellen }\end{array}$ & \multirow[t]{6}{*}{$\begin{array}{l}\text { Der Nutzen von MT bei Patienten mit einem } \\
\text { Alter von über } 90 \text { Jahren ist nicht klar }\end{array}$} & \multirow{6}{*}{$\begin{array}{l}\text { Wir empfehlen die MT auch bei } \\
\text { LVO-Patienten mit einem Alter von über } \\
90 \text { Jahren, sofern sie vorher selbstständig } \\
\text { waren }\end{array}$} \\
\hline & Grad der Evidenz: moderat $\oplus \oplus \oplus$ & & \\
\hline & Stärke der Empfehlung: stark $\uparrow$ & & \\
\hline & $\begin{array}{l}\text { ESO/ESMINT schlägt vor auch ältere } \\
\text { LVO-Patienten (> } 80 \text { Jahre) im Zeitfens- } \\
\text { ter von } 6-24 \mathrm{~h} \text { zu behandeln, sofern sie die } \\
\text { DAWN- und DEFUSE-3-Kriterien erfüllen }\end{array}$ & & \\
\hline & Grad der Evidenz: niedrig $\oplus \oplus$ & & \\
\hline & Stärke der Empfehlung: schwach $\uparrow ?$ & & \\
\hline \multirow[t]{3}{*}{$\begin{array}{l}\text { Vorbesteh- } \\
\text { ende Defizi- } \\
\text { te (prämor- } \\
\text { bider mRS } \\
>1 \text { ) }\end{array}$} & \multirow[t]{3}{*}{ Keine spezifische Empfehlung } & $\begin{array}{l}\text { Auch wenn der Nutzen unklar ist, könnte bei } \\
\text { Patienten mit einem proximalen Verschluss der } \\
\text { vorderen Zirkulation eine MT auch bei einem } \\
\text { prämorbiden mRS }>1 \text { in einem Zeitfenster von } \\
6 \text { h durchgeführt werden }\end{array}$ & \multirow{3}{*}{$\begin{array}{l}\text { Aufgrund der geringen Risiken der MT soll- } \\
\text { ten vorbestehende Defizite nicht zwingend } \\
\text { ein Ausschlussgrund für die MT sein. Wichti- } \\
\text { ger noch sollte das Ermitteln des prämorbi- } \\
\text { den mRS die Therapie nicht verzögern } \\
\text { Wir empfehlen ein pragmatisches Kriterium, } \\
\text { wie z. B., ob der Patient aus dem eigenen } \\
\text { Haushalt kommt oder aus einem Pflegeheim }\end{array}$} \\
\hline & & Grad der Evidenz: B-R & \\
\hline & & Stärke der Empfehlung: schwach $\uparrow ?$ & \\
\hline \multirow[t]{3}{*}{$\begin{array}{l}\text { Pre-hospi- } \\
\text { tal-Skalen } \\
\text { für die } \\
\text { Erkennung } \\
\text { von LVOs }\end{array}$} & $\begin{array}{l}\text { Keine spezifische Empfehlung. Patienten } \\
\text { sollten, wenn möglich, in RCTs einge- } \\
\text { schlossen werden }\end{array}$ & $\begin{array}{l}\text { Es sollten effektive Pre-hospital-Skalen entwi- } \\
\text { ckelt werden, um Patienten mit einer hohen } \\
\text { Wahrscheinlichkeit für einen LVO zu identifi- } \\
\text { zieren, um den Transport der Patienten in ein } \\
\text { MT-fähiges Krankenhaus zu beschleunigen }\end{array}$ & \multirow[t]{3}{*}{$\begin{array}{l}\text { Pre-hospital-Skalen wie z. B. RACE oder Tele- } \\
\text { konsultation könnten die Triage von vermu- } \\
\text { teten LVO-Patienten verbessern. Sie sollten } \\
\text { in Zusammenarbeit mit den Notdiensten } \\
\text { evaluiert werden }\end{array}$} \\
\hline & Grad der Evidenz: sehr niedrig $\oplus$ & Grad der Evidenz: C-EO & \\
\hline & Stärke der Empfehlung: neutral - & Stärke der Empfehlung: stark $\uparrow \uparrow$ & \\
\hline
\end{tabular}

Verschluss der häufigste Grund dafür, keine MT durchzuführen, selbst beim Nachweis einer großen, potenziell rettbaren Penumbra [16]. Große Registerstudien (z. B. PLUMBER, STOP Stroke und INTERRSeCT Register) legen nahe, dass bei ca. 20-25\% aller AIS-Patienten ein Verschluss vorliegt, der nicht unter die oben genannte Definition des LVO fällt $[17,18]$. Die Datenlage für diese sog. „medium vessel occlusions“ (MeVO, definiert als Verschluss des M2-, M3- oder M4-Segments der A. cerebri media, des A1-, A2- oder A3-Segments der A. cerebri anterior sowie des P1und P2-Segments der A. cerebri posterior) ist trotz des häufigen Auftretens unzureichend [19].

Es wurden allerdings nur wenige (ca. 10\% der Studienpopulationen) $\mathrm{Pa}$ - tienten mit einem Verschluss des M2Segments in die bisher durchgeführten RCTs eingeschlossen, entweder aufgrund einer primär falschen Klassifikation als M1 $(n=71)$ oder da ein Einschluss laut Protokoll ausdrücklich erlaubt war $(n=59)$. In einer Metaanalyse dieser RCTs konnte bei einer Stichprobe von insgesamt 130 Patienten (67 MT-Patienten) gezeigt werden, dass 


\begin{tabular}{|c|c|c|c|}
\hline Indikation & ESO/ESMINT-Guidelines [6] & AHA/ASA-Guidelines [5] & $\begin{array}{l}\text { Aktuelle Forschungsergebnisse und } \\
\text { Empfehlung der Autoren }\end{array}$ \\
\hline \multirow[t]{3}{*}{$\begin{array}{l}\text { Ziel der } \\
\text { Throm- } \\
\text { bektomie }\end{array}$} & $\begin{array}{l}\text { Bei Patienten mit LVO sollte versucht } \\
\text { werden, } \mathrm{mTICI} 3 \text { zu erreichen, sofern es } \\
\text { möglich ist mit vertretbarer Sicherheit }\end{array}$ & $\begin{array}{l}\text { Das technische Ziel der MT sollte eine Repe } \\
\text { sion vom Grad } \mathrm{mTICI} \geq 2 \text { b sein }\end{array}$ & \multirow{3}{*}{$\begin{array}{l}\text { Wir empfehlen als Ziel der MT eine voll- } \\
\text { ständige bzw. fast vollständige (mTICI 2c/3) } \\
\text { Reperfusion. Wir empfehlen wiederhol- } \\
\text { te Manöver auch bei bereits erreichter } \\
\text { mTICl-2b-Reperfusion, wenn es technisch } \\
\text { sicher möglich ist und das noch nichtperfun- } \\
\text { dierte Areal funktionell wichtig ist }\end{array}$} \\
\hline & Grad der Evidenz: niedrig $\oplus \oplus$ & Grad der Evidenz: $A$ & \\
\hline & Stärke der Empfehlung: stark $\uparrow$ & Stärke der Empfehlung: stark $\uparrow \uparrow$ & \\
\hline \multicolumn{4}{|c|}{ 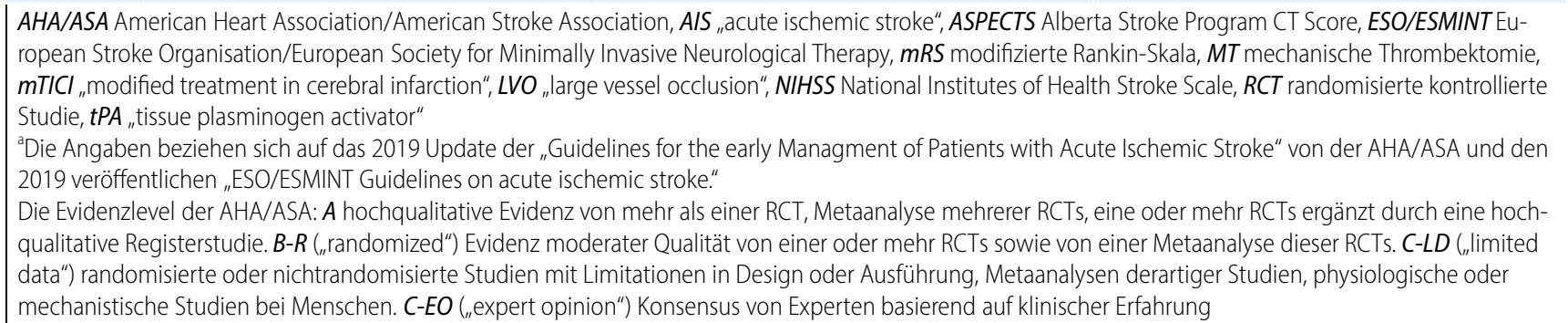 } \\
\hline
\end{tabular}

die MT signifikant häufiger zu einem guten klinischen Ergebnis (definiert als mRS $\leq 2$ an Tag 90) geführt hat als ein rein medikamentöses Management (absoluter Unterschied 18,5\%; $p=0,04$ ) [20]. Aufgrund von Bedenken bezüglich der externen Validität dieser Metaanalyse wurden die Guidelines hinsichtlich der Behandlung des M2-Verschlusses aber weder von der ASA noch von der ESO angepasst $[5,21]$.

Für andere MeVOs gibt es aktuell keine Daten aus RCTs und die Ergebnisse aus retrospektiven Studien und Registern sind teilweise widersprüchlich, wobei sich in den meisten Studien kein Hinweis auf eine erhöhte Mortalität oder ein erhöhtes Risiko an Blutungen durch die MT ableiten lässt [22, 23]. Eine kürzlich veröffentliche Studie mit der bisher höchsten Anzahl an Patienten mit isoliertem P2- und P3-Verschluss $(n=243)$ lässt aber zu mindestens in der Subgruppe der schwer betroffenen (National Institute of Health Stroke Scale [NIHSS] $\geq 10$ ) Patienten einen klinischen Effekt erwarten [24].

\section{) Nicht nur der Gefäßabschnitt, sondern auch das betroffene Hirnareal sollte betrachtet werden}

Das Problem der Evaluierung von MT bei MeVOs ist auch, dass sich die von ihnen perfundierten Hirnareale hinsichtlich ihrer Größe, aber auch Funktion deutlich unterscheiden [25]. Daher sollte nicht nur der Gefäßabschnitt betrachtet werden, sondern auch welches Hirnareal betroffen ist und wie schwer die neurologischen Ausfälle sind. Insgesamt gesehen sollte die Durchführung von RCTs zu dieser Fragestellung ein ausgesprochen wichtiges Anliegen sein, da ca. 50\% der Patienten mit einem MeVO auch trotz intravenöser Thrombolyse (i.v. „tissue plasminogen activator" [tPA]) eine bleibende relevante neurologische Behinderung entwickeln [26, 27].

Wir empfehlen bei M2-Verschlüssen die großzügige Durchführung der MT, sofern ein klinischer Vorteil für den Patienten zu erwarten ist. Bei anderen MeVOs sollte eine MT erwogen werden, sofern der Verschluss sicher erreichbar ist und das betroffene Areal von hoher funktioneller Bedeutung ist.

\section{Milde Schlaganfälle}

Der NIHSS ist nicht nur wesentlich für die Diagnose eines Schlaganfalls, sondern auch für die weitere Therapieentscheidung [5]. Während für die i.v. tPA ein NIHSS $\geq 4$ als Kriterium genutzt wird, ist bei der MT als Grenze ein NIHSS von $\geq 6$ weitgehend aktzeptiert [5]. Interessanterweise waren die NIHSS-Grenzen in den bisherigen RCTs sehr variable. So war die Grenze in der MR-CLEANStudie 2, während die ESCAPE- und
EXTEND-IA-Studien gar keine NIHSSGrenze nutzten, aber „behindernde“ Symptome vorrausetzten [28-30]. Da aber verschiedene Studien, unter anderem die SWIFT-PRIME-, REVASCATund DEFUSE-3-Studie, unabhängig eine NIHSS-Grenze von 6 validierten, wurde diese in späteren Guidelines genutzt [5].

Zur Wirksamkeit der MT bei Patienten mit einem NIHSS $<6$ gibt es kaum randomisiert-kontrollierte Daten. Retrospektive Daten aus dem Schweizer Schlaganfallregister $(n=312)$ sowie mehrere Multicenterstudien legen allerdings nahe, dass kein Zusatznutzen der MT gegenüber der alleinige i.v. tPA in dieser Patientengruppe besteht. Es gab aber auch keine Hinweise auf ein erhöhtes Komplikationsrisiko [31-33].

Den oben genannten Studien widersprechend weisen Daten der MINORSTROKE-Kollaboration ( $n=598$ Patienten) darauf hin, dass milde betroffene Patienten mit einer M1-Okklusion von einer zusätzlichem MT profitieren könnten, während bei einer M2-Okklusion die alleinige i.v. tPA überlegen sein könnte [34].

\section{》) Bei milden Schlaganfällen sollte die Therapieentscheidung sorgfältig abgewogen werden}

Bei der Betrachtung dieser Studienergebnisse muss neben dem retrospektiven Charakter und möglichen Selektionsbias 
aber auch auf die inhärenten Limitationen des NIHSS hingewiesen werden. Der NIHSS bildet nicht das Ausmaß der individuellen Behinderung durch die neurologischen Defizite ab. So würde eine Dysarthrie im NIHSS nur einem Punktwert von 1 entsprechen, aber im Falle einer Persistenz bei Lehrern und Anwälten $\mathrm{zu}$ gravierenden Langzeitfolgen führen. Ähnliches gilt für eine Schwäche der Hand bei einem Musiker oder Feinmechaniker. Basierend auf diesen Gedankengängen kann bei milden Schlaganfällen keine allgemeine Empfehlung ausgesprochen werden und die Therapieentscheidung sollte nach sorgfältiger Abwägung des möglichen Nutzens und der Risiken getroffen werden. Da Patienten mit einem NIHSS $<6$ meistens noch kommunizieren können, sollte diese Entscheidung idealerweise in Absprache mit dem Patienten getroffen werden.

\section{Verschluss der A. basilaris}

Verschiedene Metaanalysen retrospektiver Studien legen nahe, dass die MT auch bei Verschlüssen der A. basilaris gegenüber einer rein medikamentösen Therapie zu einem verbesserten langfristigen neurologischen Ergebnis führt, ohne dabei mit erhöhten Komplikationsraten einherzugehen $[35,36]$. $\mathrm{Zu}$ diesem Ergebnis kam auch eine jüngst veröffentliche Registerstudie mit 829 Patienten. In dieser Studie erhöhte die MT die Wahrscheinlichkeit für ein gutes klinisches Ergebnis $(m R S \leq 3)$ deutlich [37]. Dem gegenüber stehen Ergebnisse aus zwei RCTs (BEST und BASICS), die keinen signifikanten positiven Effekt der MT auf das klinische Ergebnis bei Verschlüssen der A. basilaris zeigen konnten $[38,39]$. Es muss allerdings beachtet werden, dass in beiden Studien initial heute nicht mehr genutzte Katheterverwendet wurden und das es aufgrund zwischenzeitlich neu verfügbarer Daten zu hohen Cross-over-Raten (von konservativ zu MT) und verminderter Rekrutierung (Die BEST-Studie wurde früher als geplant beendet) kam $[38,39]$. Daher kann aus diesen Studien keine definitive Empfehlung abgeleitet werden, vor allem bei schwer betroffenen Patienten mit kleinem Infarktkern.
Leider ist auch für die Zukunft nicht zu erwarten, dass diese Fragestellung durch eine RCT geklärt wird, da aufgrund des starken Effekts der MT bei anderen Verschlüssen starker Widerstand gegen eine Randomisierung zu erwarten ist. Aus diesem Grund und auf Basis unserer klinischen Erfahrung empfehlen wir im Kontrast zu den zwei RCTs die MT für Verschlüsse des vertebrobasilaren Systems, vor allem bei schwer betroffenen Patienten mit kleinem Infarktkern.

\section{Tandem-Verschlüsse}

Unter einem Tandem-Verschluss versteht man die Kombination aus einem intrakraniellen Verschluss (z. B. des M1-Segments) und einer/eines extrakraniellen Stenose/Verschlusses (z. B. der A. carotis interna $[\mathrm{ACI}]$ ) im gleichen Stromgebiet. Diese Verschlüsse sind technisch schwerer zu behandeln und das klinische Ergebnis ist meistens schlechter als bei isolierten Verschlüssen [40].

In der HERMES-Analyse wurden 122 Patienten (von 1254) mit einem Tandem-Verschluss eingeschlossen, es wurde aber keine Subgruppenanalyse für diese Patientengruppe durchgeführt [1]. Weiterhin war die Behandlung des extrakraniellen Verschlusses nicht standardisiert und variierte (keine Rekanalisation, Angioplastie oder Stenting). Eine große retrospektive Multicenterstudie ( $n=482$ Patienten), durchgeführt von der „Thrombectomy-in-TandemLesions"(TITAN)-Gruppe, legt nahe, dass primäres Stenting der extrakraniellen Läsion die beste Chance auf eine erfolgreiche Rekanalisation beider Verschlüsse bietet (mTICI [„modified treatment in cerebral infarction" $] \geq 2 \mathrm{~b}$ Rate von $79,4 \%$ gegenüber nur $60,2 \%$ bei reiner MT; [41]). Es konnte zwar kein signifikanter Effekt auf das klinische Ergebnis gezeigt werden, das Komplikationsrisiko war aber ebenfalls nicht erhöht [42]. Aufgrund des Mangels an hochqualitativen Daten hinsichtlich der optimalen Behandlungsstrategie für Tandem-Verschlüsse planen die TITANKollaborateure, eine RCT zu starten [43].

Basierend auf diesen vorläufigen $\mathrm{Da}$ ten sowie vielversprechenden neuen Techniken für die Behandlung von Tan-
dem-Verschlüssen [44] empfehlen wir die Durchführung einer MT bei Tandem-Verschlüssen mit intrakraniellem LVO sowie bei ausgewählten Patienten das primäre Stenting der extrakraniellen Läsion.

\section{Pädiatrische Schlaganfälle (0-17 Jahre)}

Auch wenn der AIS mit einer Inzidenz von nur ca. 2 bis 8 pro 100.000 Kindern pro Jahr ein seltenes Ereignis ist, ist er dennoch ein wesentlicher Grund für langfristige Behinderungen und den Verlust an Lebensjahren [45]. Bis vor kurzem war die Evidenz für die MT in pädiatrischen Populationen (0-17 Jahre) auf kleine Fallserien beschränkt. Dies hat sich 2020 durch die Publikation zweier Arbeiten verändert, einer Metaanalyse basierend auf individuellen Patientendaten $(n=110)$ sowie der retrospektiven Multicenterstudie Save ChildS $(n=73)$. Beide Studien zeigen übereinstimmend ermutigende Daten sowohl hinsichtlich des angiographischen Erfolges (jeweils mTICI $\geq 2$ b-Raten von über $80 \%$ ) als auch hinsichtlich des klinischen Ergebnisses bei einem vertretbaren Sicherheitsprofil [46, 47]. Eine sekundäre Analyse der Save ChildS Daten zeigte weiterhin, dass die klinisch neurologischen Ergebnisse generell gut sind unabhängig von Ätiologie, Alter und damit verbundener MTTechnik [48]. Weiterhin konnte gezeigt werden, dass analog zu Erwachsenen die MT auch bei pädiatrischen Patienten im erweiterten Zeitfenster (6-24h) zu einem guten neurologischen Ergebnis führt, sofern ein relevantes Mismatch zwischen Infarktkern und Penumbra besteht [49].

Auch wenn die genannten Studien nicht an die Qualität von RCTs heranreichen, werden in absehbarer Zeit keine Daten höherer Qualität verfügbar sein. Unterstrichen wird diese Annahme durch das Scheitern der Thrombolysisin-Pediatric-Stroke(TIPS)-Studie, die aufgrund fehlender Patientenrekrutierung nach über einem Jahr Studiendauer und 14 aktivierten Zentren abgebrochen wurde. Ähnliches wäre bei einer RCT zur MT bei pädiatrischen AIS-Patienten zu erwarten, da in Anbetracht des sehr großen Effektes bei erwachsenen Patien- 
ten kaum Ärzte und auch Eltern bereit wären, Kinder zu randomisieren.

Zusammenfassend empfehlen wir insbesondere aufgrund des günstig erscheinenden Risikoprofils die MT bei pädiatrischen Patienten mit LVO, selbst im verlängerten Zeitfenster von 6-24h. Vorsicht ist aber bei einem begründeten Verdacht auf eine Arteriopathie angebracht.

\section{MT bei sehr alten Patienten (>90 Jahre)}

Die aktuelle AHA/ASA-Guideline gibt keine genaue Altersgrenze nach oben für die Durchführung der MT vor, allerdings fehlen bei Patienten mit einem Alter von über 90 Jahren randomisierte Daten und so kann aktuell keine abschließende Aussage zur Wirksamkeit der MT bei Patienten in diesem Alter getroffen werden [5]. Nichtrandomisierte Studien legen allerdings einen positiven Effekt der MT auch in dieser Patientengruppe nahe [50].

\section{》) Der Großteil der Ärzte entscheidet sich auch bei Patienten > 90 Jahren für eine MT}

So zeigte sich in einer Analyse von 124 über 90 Jahre alten Patienten aus dem ETIS-Register, dass die Chancen auf ein gutes neurologisches Ergebnis (definiert als $\mathrm{mRS} \leq 3$ nach 90 Tagen) signifikant höher waren, sofern eine erfolgreiche Rekanalisation erreicht wurde [51]. Weiterhin konnte in bisherigen Analysen kein erhöhtes Risiko für Komplikationen in dieser Altersgruppe nachgewiesen werden $[1,51]$. Passend dazu zeigte eine Umfrage unter 607 Neuroradiologen und Neurologen, dass der Großteil (74\%) sich auch bei Patienten über 90 Jahren für eine MT entscheidet, sofern eine klare Indikation (Level 1A) vorliegt [52].

Zusammenfassend sollte das Alter aus unserer Sicht kein Ausschlussgrund für die MT sein, insbesondere bei Patienten, die vor dem Schlaganfall eine gute Lebensqualität hatten.
Patienten mit vorbestehenden Behinderungen (mRS $>1$ )

Aufgrund der Einschlusskriterien der bisher durchgeführten RCTs gibt es bisher kaum Daten zum Effekt der MT bei Patienten mit einem mRS $>1$. So war nur in der MR-CLEAN-Studie ein mRS $>1$ kein Ausschlusskriterium für die MT und es wurden 21 Patienten mit vorbestehender Behinderung eingeschlossen [28]. Da diese Patienten allerdings nicht gesondert analysiert wurden, lässt sich aktuell keine Aussage über diese Patientengruppe treffen. Dies ist insbesondere problematisch, da Patienten mit einem mRS >1 ca. $30 \%$ aller Patienten mit einem LVO ausmachen [53]. Kleinere retrospektive Studien legen allerdings nahe, dass bei ungefähr einem Drittel der prämorbide Status erhalten werden kann, was dem Ziel der MT entspricht [54]. Dies konnte jüngst von Salwi et al. in einer größeren Kohorte $(n=259)$ bestätigt werden. Insgesamt konnten sie dabei keine ausreichenden Belege dafür finden, dass sich das klinische Ergebnis von Patienten mit prämorbider moderater Behinderung (mRS 2-3) von Patienten mit leichter bis keine Behinderung ( $\mathrm{mRS}$ 0-1) unterscheidet [55].

Wir empfehlen daher, dass das Erheben des prämorbiden Status für die Therapieentscheidung eine untergeordnete Rolle spielen sollte und in keinem Fall zu einer Verzögerung der MT führen darf, da der Effekt der MT durch intrahospitale Zeitverzögerungen dramatisch abnimmt [56]. Hier wäre ein pragmatischer Ansatz als Kriterium den Lebensort des Patienten zu nutzen, z. B. kommt aus eigenem Haushalt vs. Pflegeheim.

\section{Neue Entwicklung in Behandlung und Management von LVO-Patienten}

\section{Intravenöse Thrombolyse bei MT-Patienten}

Die Zulassung der i.v. tPA erfolgte 1995, nachdem ein deutlich verbessertes klinisches Ergebnis durch i.v. tPA bei AISPatienten im Zeitfenster von $3 \mathrm{~h}$ nach Symptombeginn gezeigt werden konnten. Durch spätere Studien konnte die- ses Zeitfenster auf 4,5 h erweitert werden und auch bei ausgewählten AIS-Patienten bei unbekanntem Zeitfenster ist die i.v. tPA bei passender Bildgebung mittlerweile indiziert $[57,58]$. Durch diesen historischen Zusammenhang wurde die MT bisher in RCTs nur als Zusatz zur i.v. tPA evaluiert [1]. Allerdings weisen neuere nichtrandomisierte Daten darauf hin, dass die i.v. tPA bei MT-fähigen Patienten keinen positiven Zusatznutzen haben könnte bzw. sogar das Blutungsrisiko erhöhen könnte [59]. Es gibt allerdings auch Daten, die auf einen möglichen positiven Effekt der i.v. TPA in diesem Patientenkollektiv hinweisen [60]. Erschwert wird die Bewertung der Effektivität der i.v. tPA durch den Umstand, dass die i.v. tPA je nach Thrombuslokalisation und Thrombuszusammensetzung unterschiedlich effektiv ist [61]. Interessanterweise kann auch nicht von einer Verbesserung der MT-Ergebnisse durch Weglassen der i.v. tPA auf ein besseres klinisches Ergebnis geschlossen werden. Daten aus zwei multizentrischen Kohorten weisen sogar daraufhin, dass das klinische Ergebnis bei Patienten nach Kombinationstherapie besser ist, obwohl das finale MT-Ergebnis schlechter war [62, 63]. Diese Daten sind wichtig, da das primäre Ziel einer Therapie immer das klinische Ergebnis sein muss und kein Surrogatparameter, wie das Reperfusionsergebnis.

Die beste derzeit verfügbare Evidenz stammt aus drei kürzlich veröffentlichen RCTs. Diese haben untersucht, ob die alleinige MT der Kombinationstherapie hinsichtlich des klinischen Ergebnisses (mRS nach 90 Tagen) nicht unterlegen ist. Während dies die in Japan durchgeführte SKIP-Studie nicht zeigen konnte [64], war die alleinige MT der Kombinationstherapie in den zwei chinesischen Studien entsprechend der vordefinierten Kriterien (DIRECT-MT [65], DEVT [66]) nicht unterlegen. Aufgrund der widersprüchlichen Ergebnissen, deutlich verlängerten Zeitspannen (z.B. Dauer von 59 min zwischen Patientenankunft und Bolusgabe) in den chinesischen Studien sowie auch abweichenden Schlaganfallätiologien in asiatischen Populationen haben die amerikanischen und europäischen Fachgesellschaften ihre Guide- 
lines nicht angepasst [67]. Aktuell werden in Europa zwei RCTs mit dieser Fragestellung durchgeführt: SWIFT DIRECT (NCT03192332) und MR CLEAN-NOIV (ISRCTN80619088). Es gilt allerdings, dass die oben genannten Überlegungen nur für Patienten gelten, die sich direkt im MT-fähigen Krankenhaus vorstellen.

\section{1) Die iv. tPA-Gabe sollte auf keinen Fall die MT verzögern}

Ein weiterer wesentlicher Unsicherheitsfaktor ist, ob die Studienergebnisse auch auf die bisher nicht standardmäßig verwendete Tenecteplase übertragbar sind, da diese laut einer kürzlich veröffentlichen RCT der Alteplase hinsichtlich früher Rekanalisation sowie klinischem Ergebnis überlegen ist [68]. Weiterhin ist Tenecteplase auch aufgrund der Möglichkeit zur Einmalgabe als Bolus Alteplase überlegen [68].

Bis zum Ergebnis dieser Studien empfehlen wir weiterhin in Übereinstimmung mit aktuellen Guidelines die kombinierte Therapie bei geeigneten $\mathrm{Pa}$ tienten, ggf. sollte aber bei bestehender Zulassung auf Tenecteplase gewechselt werden. Die iv. tPA-Gabe sollte aber auf keinen Fall die MT verzögern.

\section{Neue Triage-Konzepte}

Bei AIS-Patienten ist die Zeit zwischen Symptom- und Behandlungsbeginn von herausragender Bedeutung, allerdings besteht je nach Ätiologie des AIS (MT geeignet vs. nur Lysetherapie geeignet) ein Zielkonflikt im Hinblick auf die Triage vor der Krankenhauseinlieferung [69]. Während ein AIS-Patient mit einem Verschluss, der für die MT nicht zugänglich ist, in das nächste lysefähige Krankenhaus transferiert werden sollte, ist für einen Patienten mit LVO ein Transfer in das nächstgelegene MT-fähige Krankenhaus von Vorteil. Dies gilt auch, wenn die primäre Transportzeit bis zu 30 min länger ist [70].

Aus diesem Grund haben verschiedene Autorengruppen Skalen für die Detektion von LVOs entwickelt. Wesentlich hier zu nennen sind die RapidArterial-Occlusion-Evaluation(RACE)-
Skala, der Los Angeles Motor Score (LAMS), die Gaze-Face-Arm-SpeechTime(G-FAST)-Skala und die Field-Assessment-Stroke-Triage-for-EmergencyDepartment(FAST-ED)-Skala [71]. Zwei kürzlich veröffentliche prospektive, multizentrische Studien mit jeweils über 1000 Patienten konnten für jede dieser Skalen eine akzeptable bis gute Genauigkeit zeigen, wobei in beiden Studien die RACESkala die höchste Genauigkeit hatte [71, 72]. Insgesamt zeigt sich aber weiterhin, dass der von Ärzten erhobene NIHSS die höchste Genauigkeit hat [71]. Eine Kombination beider Techniken durch Telekonsultation im Krankenwagen wurde daher in Stockholm erprobt und hat dort zu deutlich verkürzten Transferzeiten geführt sowohl für die i.v. tPA als auch für die MT [73]. Eine Gruppe des Universitätsspitals Basel evaluiert derzeit einen ähnlichen Ansatz in der Telestroke2-Studie (NCT04578002). Eine weitere technische Möglichkeit zur optimalen Triage von AIS-Patienten außerhalb des Krankenhauses ist die Nutzung von „mobile stroke units“. Für diese konnte in einer Metaanalyse bereits eine deutlich reduzierte Zeit bis zum Behandlungsbeginn gezeigt werden [74]. Allerdings bestehen aufgrund der hohen Kosten noch erhebliche Widerstände gegen eine breite Einführung.

\section{I) Das OneStop-Manage- ment führt zu erheblichen Zeitersparnissen}

Zur Minimierung der Zeit von der Krankenhaustür bis zum Beginn der MT wurden ebenfalls neue Triage- und Workflow-Konzepte entwickelt. Ein wesentlicher Ansatz dafür ist das OneStopManagement, bei dem der Patient direkt in die Angiographieeinheit verbracht wird und dort die primäre Bildgebung stattfindet [75]. Dadurch wird ein „stop“" im Multidetector-CT(MDCT)- bzw. MRT-Raum eingespart. Untersuchungen zeigen, dass in vielen Krankenhäusern erhebliche Distanzen zwischen dem CT/MRT- und dem Angiographieraum besteht [76]. Aber selbst in Krankenhäusern, bei denen Angiographie- und MDCT-Raum nebeneinander sind, führt das OneStop-Management zu erheblichen Zeitersparnissen (Median $25 \mathrm{~min}$ [Interquartilsabstand (IQR) 19-30] vs. Median $60 \mathrm{~min}$ [IQR 48-68]; $p<0,001$; [77]).

Zusammenfassend empfehlen wir die Einführung einfach durchzuführender Pre-hospital-Scores wie z. B. der RACESkala, um eine verbesserte Triage von AIS-Patienten zu erreichen.

\section{Technisches Ziel der MT}

Allgemein wird eine MT als „erfolgreich“ definiert, sofern am Ende der Prozedur ein mTICI von mehr als 2 b erreicht wurde, dies entspricht einer Reperfusion von mehr als $50 \%$ des vorher nichtperfundierten Areals [78]. Aufgrund technischer Fortschritte und der Erkenntnis, dass die Reperfusion einer der wichtigsten Einflussfaktoren für das langfristige klinische Ergebnis ist, wurde von verschiedenen Autorengruppen vorgeschlagen, dass ein mTICI-Score von $2 c$ oder 3 (Perfusion von $\geq 90 \%$ des vorher nichtperfundierten Areals) als neue Definition genutzt werden sollte $[79,80]$. Es konnte in mehreren retrospektiven Studien gezeigt werden, dass eine sekundäre Verbesserung des mTICI $2 b$ auf $2 c / 3 \mathrm{zu}$ einer Verbesserung des klinischen Ergebnisses führt [81]. Dies gilt auch für den Fall, dass sich die Prozedur dadurch verlängert [82]. Allerdings muss hier auf einen möglichen Selektionsbias hingewiesen werden, da aus den Arbeiten nicht klarhervorgeht, warum bei einem Teil der Patienten eine Verbesserung des MT-Ergebnisses angestrebt wurde und bei den anderen Patienten nicht. Insgesamt wären zu dieser Fragestellung RCTs wünschenswert, um abschließend zu klären, ob eine Verbesserung des MT-Ergebnisses tatsächlich zu klinisch besseren Ergebnissen führt oder ob sie mit einem erhöhten Komplikationsrisiko einhergeht.

Zusammenfassend empfehlen wir aktuell die Verbesserung des angiographischen Ergebnisses bei ausgewählten $\mathrm{Pa}$ tienten, hierbei sollten in Übereinstimmung mit einem kürzlich erschienen Expertenstatement primär folgende Fragestellungen eine Rolle spielen:

- Ist das nichtperfundierte Areal bereits infarziert? 
- Welche funktionelle Bedeutung hat es?

- Wie hoch sind die möglichen Risiken bei einem weiteren MT-Manöver [83]?

\section{Fazit für die Praxis}

\section{- Zusammenfassend empfehlen wir die mechanische Thrombektomie (MT) unabhängig vom Zeitfenster zu erwägen, auch wenn die DAWN- und DEFUSE-3-Kriterien nicht erfüllt sind, sofern noch ausreichend Penumbra nachweisbar ist. \\ - Zusätzlich sollten auch Patienten mit einem Verschluss der A. basilaris oder einem Tandem-Verschluss nicht von der MT ausgeschlossen werden. Dies gilt unserer Einschätzung nach auch für sehr alte (>90 Jahre) und junge (0-17 Jahre) Patienten. \\ - Bei Patienten mit vorbestehender Behinderung oder bereits sehr aus- gedehnten Infarktzeichen sollte die MT weiterhin eine Einzelfallentschei- dung darstellen. \\ - Für die Zukunft gehen wir davon aus, dass sich die Indikation für eine MT aufgrund technischer Verbes- serungen deutlich erweitern wird, vor allem auf distal gelegene, kleine Verschlüsse.}

\section{Korrespondenzadresse}

\section{Prof. Dr. Marios-Nikos Psychogios}

Abteilung für interventionelle und diagnostische Neuroradiologie, Klinik für Radiologie und Nuklearmedizin, Universitätsspital Basel

Petersgraben 4, 4031 Basel, Schweiz marios.psychogios@usb.ch

Funding. Open access funding provided by University of Basel

\section{Einhaltung ethischer Richtlinien}

Interessenkonflikt. M.-N. Psychogios, A. Brehm, P. Sporns und L.H. Bonati geben an, dass kein Interessenkonflikt besteht.

Für diesen Beitrag wurden von den Autoren keine Studien an Menschen oder Tieren durchgeführt. Für die aufgeführten Studien gelten die jeweils dort angegebenen ethischen Richtlinien.
Open Access. Dieser Artikel wird unter der Creative Commons Namensnennung 4.0 International Lizenz veröffentlicht, welche die Nutzung, Vervielfältigung, Bearbeitung, Verbreitung und Wiedergabe in jeglichem Medium und Format erlaubt, sofern Sie den/die ursprünglichen Autor(en) und die Quelle ordnungsgemäß nennen, einen Link zur Creative Commons Lizenz beifügen und angeben, ob Änderungen vorgenommen wurden.

Die in diesem Artikel enthaltenen Bilder und sonstiges Drittmaterial unterliegen ebenfalls der genannten Creative Commons Lizenz, sofern sich aus der Abbildungslegende nichts anderes ergibt. Sofern das betreffende Material nicht unter der genannten Creative Commons Lizenz steht und die betreffende Handlung nicht nach gesetzlichen Vorschriften erlaubt ist, ist für die oben aufgeführten Weiterverwendungen des Materials die Einwilligung des jeweiligen Rechteinhabers einzuholen.

Weitere Details zur Lizenz entnehmen Sie bitte der Lizenzinformation auf http://creativecommons.org/ licenses/by/4.0/deed.de.

\section{Literatur}

1. Goyal M, Menon BK, Van Zwam WH, Dippel DWJ, Mitchell PJ, DemchukAM et al (2016) Endovascular thrombectomy after large-vessel ischaemic stroke: A meta-analysis of individual patient data from five randomised trials. Lancet 387:1723-1731

2. Tsivgoulis G, Goyal N, Mikulik R, Sharma VK, Katsanos AH, Zand R et al (2016) Eligibility for mechanical thrombectomy in acute ischemic stroke: A phase iv multi-center screening log registry. J Neurol Sci 371:96-99

3. Rai AT, Seldon AE, Boo S, Link PS, Domico JR, Tarabishy AR et al (2017) A population-based incidence of acute large vessel occlusions and thrombectomy eligible patients indicates significant potential for growth of endovascular stroke therapy in the USA. J Neurolntervent Surg 9:722

4. Vanacker $P$, Lambrou D, Eskandari A, Mosimann PJ Maghraoui A, Michel P (2016) Eligibility and predictors for acute revascularization procedures in a stroke center. Stroke 47:1844-1849

5. Powers William J, Rabinstein AA, Ackerson T, Adeoye Opeolu M, Bambakidis NC, Becker K et al (2019) Guidelines for the early management of patients with acute ischemic stroke: 2019 update to the 2018 guidelines for the early management of acute ischemic stroke: A guideline for healthcare professionals from the american heart association/ american stroke association. Stroke 50:e344-e418

6. Turc G, Bhogal P, Fischer U, Khatri P, Lobotesis K, Mazighi M et al (2019) European stroke organisation (eso)-european society for minimally invasive neurological therapy (esmint) guidelines on mechanical thrombectomy in acute ischemic stroke. J Neurolntervent Surg. https://doi.org/10. 1136/neurintsurg-2018-014569

7. Nogueira RG, Jadhav AP, Haussen DC, Bonafe A, Budzik RF, Bhuva P et al (2018) Thrombectomy 6 to 24 hours after stroke with a mismatch between deficit and infarct. N Engl J Med 378:11-21

8. Albers GW, Marks MP, KempS, Christensen S, Tsai JP, Ortega-Gutierrez S et al (2018) Thrombectomy for stroke at 6 to 16 hours with selection by perfusion imaging. N Engl J Med 378:708-718
9. Desai SM, Rocha M, Molyneaux BJ, Starr M, Kenmuir CL, Gross BA et al (2018) Thrombectomy 6-24 hours after stroke in trial ineligible patients. JNeurolntervent Surg 10:1033-1037

10. Siegler JE, Messé SR, Sucharew H, Kasner SE, Mehta T, Arora N et al (2020) Thrombectomy in dawn- and defuse-3-ineligible patients: $\mathrm{A}$ subgroup analysis from the best prospective cohort study. Neurosurgery 86:E156-e163

11. Almekhlafi MA, Kunz WG, McTaggart RA, Jayaraman MV, Najm M, Ahn SH et al (2020) Imaging triage of patients with late-window (6-24 hours) acute ischemic stroke: A comparative study using multiphase ct angiography versus ct perfusion. american JNeuroradiol 41:129-133

12. Kim BJ, Menon BK, Kim JY, Shin DW, Baik SH, Jung $C$ et al (2020) Endovascular treatment after stroke due to large vessel occlusion for patients presenting very late from time last known well. jama Neurol. https://doi.org/10.1001/jamaneurol. 2020.2804

13. Román LS, Menon BK, Blasco J, Hernández-Pérez M Dávalos A, Majoie C et al (2018) Imaging features and safety and efficacy of endovascular stroke treatment: A meta-analysis of individual patientlevel data. lancet Neurol 17:895-904

14. Cagnazzo F, Derraz I, Dargazanli C, Lefevre P-H Gascou G, Riquelme C et al (2020) Mechanical thrombectomy in patients with acute ischemic stroke and aspects $\leq 6$ : A meta-analysis. J NeuroIntervent Surg 12:350

15. Fiehler J, Cognard C, Gallitelli M, Jansen $O$, Kobayashi A, Mattle HP et al (2016) European recommendations on organisation of interventional care in acute stroke (eroicas). Eur Stroke $J$ 1:155-170

16. Bahr Hosseini M, Woolf G, Sharma LK, Hinman JD, Rao NM, Yoo B et al (2018) The frequency of substantial salvageable penumbra in thrombectomy-ineligible patients with acute stroke. JNeuroimaging 28:676-682

17. Dozois A, Hampton L, Kingston CW, Lambert G, Porcelli TJ, Sorenson D et al (2017) Plumber study (prevalence of large vessel occlusion strokes in mecklenburg county emergency response). Stroke 48:3397-3399

18. Smith WS, Lev MH, English JD, Camargo EC, Chou M, Johnston SC et al (2009) Significance of large vessel intracranial occlusion causing acute ischemic stroke and tia. Stroke 40:3834-3840

19. Goyal M, Ospel JM, Menon BK, Mevo HMD (2020) The next frontier? J Neurointerv Surg 12:545-547

20. Menon BK, Hill MD, Davalos A, Roos Y, Campbell BCV, Dippel DWJ et al (2019) Efficacy of endovascular thrombectomy in patients with $\mathrm{m} 2$ segment middle cerebral artery occlusions: Metaanalysis of data from the hermes collaboration. JNeurointerv Surg 11:1065-1069

21. Turc G, Bhogal P, Fischer U, Khatri P, Lobotesis $K_{4}$ Mazighi $M$ et al (2019) European stroke organisation (eso)_european society for minimally invasive neurological therapy (esmint) guidelines on mechanical thrombectomy in acute ischaemic strokeendorsed by stroke alliance for europe (safe). Eur Stroke J 4:6-12

22. Premat K, Bartolini B, Baronnet-Chauvet F, Shotar E, Degos V, Muresan P et al (2018) Single-center experience using the $3 \mathrm{max}$ reperfusion catheter for the treatment of acute ischemic stroke with distal arterial occlusions. Clin Neuroradio 28:553-562

23. Haussen DC, Lima A, Nogueira RG (2016) The trevo $\mathrm{xp} 3 \times 20 \mathrm{~mm}$ retriever ('baby trevo') for 
the treatment of distal intracranial occlusions. J Neurolntervent Surg 8:295

24. Meyer L, Stracke CP, Jungi N, Wallocha M, Broocks G, Sporns PB et al (2021) Thrombectomy for primary distal posterior cerebral artery occlusion stroke: The topmost study. JAMA, Neurol

25. Saver JL, Chapot R, Agid R, Hassan A, Jadhav AP, Liebeskind DS, et al. Thrombectomy for distal, medium vessel occlusions: A consensus statement on present knowledge and promising directions. Stroke. 2020;51:2872-2884

26. Ospel JM, Menon BK, DemchukAM, Almekhlafi MA, Kashani N, Mayank A et al (2020) Clinical course of acute ischemic stroke due to medium vessel occlusion with and without intravenous alteplase treatment. Stroke 51:3232-3240

27. Sarraj A, Sangha N, Hussain MS, Wisco D, Vora N, Elijovich L et al (2016) Endovascular therapy for acute ischemic stroke with occlusion of the middle cerebral artery $\mathrm{m} 2$ segment. JAMA Neurol 73:1291-1296

28. Berkhemer OA, Fransen PSS, Beumer D, van den Berg LA, Lingsma HF, Yoo AJ et al (2014) $A$ randomized trial of intraarterial treatment for acute ischemic stroke. N Engl J Med 372:11-20

29. Goyal M, Demchuk AM, Menon BK, Eesa M, Rempel JL, Thornton J et al (2015) Randomized assessment of rapid endovascular treatment of ischemic stroke. NEngl J Med 372:1019-1030

30. Campbell BCV, Mitchell PJ, Kleinig TJ, Dewey HM, Churilov L, Yassi N et al (2015) Endovascular therapy for ischemic stroke with perfusionimaging selection. N Engl J Med 372:1009-1018

31. Goyal N, Tsivgoulis G, Malhotra K, Ishfaq MF, Pandhi A, Frohler MT et al (2020) Medical management vs mechanical thrombectomy for mild strokes: An international multicenter study and systematic review and meta-analysis. jama Neurol 77:16-24

32. Manno C, Disanto G, Bianco G, Nannoni S, Heldner M, Jung $S$ et al (2019) Outcome of endovascular therapy in stroke with large vessel occlusion and mild symptoms. Neurology 93:e1618-e1626

33. Volny O, Zerna C, Tomek A, Bar M, Rocek M, Padr R et al (2020) Thrombectomy vs medical management in low nihss acute anterior circulation stroke. Neurology 95:e3364-e3372

34. Seners P, Perrin C, Lapergue B, Henon H, Debiais S, Sablot D et al (2020) Bridging therapy or iv thrombolysis in minor stroke with large vessel occlusion. Ann Neurol 88:160-169

35. Phan K, Phan S, Huo YR, Jia F, Mortimer A (2016) Outcomes of endovascular treatment of basilar artery occlusion in the stent retriever era: A systematic review and meta-analysis. JNeurolntervent Surg 8:1107

36. Gory B, Eldesouky I, Sivan-Hoffmann R, Rabilloud $M$, Ong $E$, Riva $R$, et al. Outcomes of stent retriever thrombectomy in basilar artery occlusion: An observational study and systematic review. Journal of Neurology, Neurosurgery \& Psychiat ry. 2016;87:520

37. Zi W, Qiu Z, Wu D, Li F, Liu H, Liu W et al (2020) Assessment of endovascular treatment for acute basilar artery occlusion via a nationwide prospective registry. JAMA Neurol 77:561-573

38. Liu X, Dai Q, Ye R, Zi W, Liu Y, Wang H et al (2020) Endovascular treatment versus standard medical treatment for vertebrobasilar artery occlusion (best): An open-label, randomised controlled trial. Lancet Neurol 19:115-122

39. (2020) Eso-wso 2020 joint meeting abstracts. Int J Stroke 15:3-752
40. Sallustio F, Motta C, Koch G, Pizzuto S, Campbell BC, Diomedi $M$ et al (2017) Endovascular stroke treatment of acute tandem occlusion: A singlecenter experience. JVasc Interv Radiol 28:543-549

41. Papanagiotou P, Haussen DC, Turjman F, Labreuche J, Piotin M, Kastrup A, et al. Carotid stenting with antithrombotic agents and intracranial thrombectomy leads to the highest recanalization rate in patients with acute stroke with tandem lesions. Jacc: Cardiovascular Interventions. 2018;11:1290-1299

42. Zhu F, Bracard S, Anxionnat R, Derelle AL, Tonnelet R, Liao L et al (2019) Impact of emergent cervical carotid stenting in tandem occlusion strokes treated by thrombectomy: A review of the titan collaboration. Front Neurol 10:206

43. Zhu F, Hossu G, Soudant M, Richard S, Achit H, Beguinet M et al (2020) Effect of emergent carotid stenting during endovascular therapy for acute anterior circulation stroke patients with tandem occlusion: A multicenter, randomized, clinical tria (titan) protocol. Int J Stroke. https://doi.org/10. 1177/1747493020929948

44. Maus V, Behme D, Maurer C, Tropine A, Tritt S, Berlis A et al (2020) The rewised care technique : Simultaneous treatment of atherosclerotic tandem occlusions in acute ischemic stroke. clin Neuroradiol 30:489-494

45. Goeggel Simonetti B, Cavelti A, Arnold M, Bigi S, Regényi M, Mattle HP et al (2015) Long-term outcome after arterial ischemic stroke in children and young adults. Neurology 84:1941-1947

46. Sporns PB, Sträter R, Minnerup J, Wiendl H, Hanning U, Chapot Ret al (2020) Feasibility, safety, and outcome of endovascular recanalization in childhood stroke: The save childs study. jama Neurol 77:25-34

47. Bhatia K, Kortman H, Blair C, Parker G, Brunacci D, Ang T et al (2019) Mechanical thrombectomy in pediatric stroke: Systematic review, individual patient data meta-analysis, and case series. J Neurosurg Pediatr. https://doi.org/10.3171/ 2019.5.PEDS19126

48. Sporns PB, Straeter R, Minnerup J, Wiendl H, Hanning U, Chapot R, et al. Does device selection impact recanalization rate and neurological outcome?: An analysis of the save childs study. Stroke. 2020;51:1182-1189

49. Sporns PB, Psychogios MN, Straeter R, Hanning U, Minnerup J, Chapot R, et al. Clinical diffusion mismatch to select pediatric patients for embolec tomy 6 to 24 hours after stroke: An analysis of the save childs study. Neurology. 2021;96:e343-e351

50. Meyer L, Alexandrou M, Flottmann F, DebChatterji M, Abdullayev N, Maus V et al (2020) Endovascular treatment of very elderly patients aged $\geq 90$ with acute ischemic stroke. JAHA 9:e14447

51. Drouard-de Rousiers E, Lucas L, Richard S, Consoli A, Mazighi M, Labreuche J et al (2019) Impact of reperfusion fornonagenarians treated by mechanical thrombectomy. Stroke 50:3164-3169

52. Ospel JM, Kashani NN, Menon B, Almekhlafi M, Wilson A, Fischer U et al (2020) Endovascular treatment decision making in octogenarians and nonagenarians : Insights from unmask evt an international multidisciplinary study. clin Neuroradiol 30:45-50

53. Quinn TJ, Taylor-Rowan M, Coyte A, Clark AB, Musgrave SD, Metcalf AK et al (2017) Prestroke modified rankin scale: Evaluation of validity, prognostic accuracy, and association with treatment. Front Neurol. https://doi.org/10.3389/ fneur.2017.00275
54. Slawski DE, Salahuddin H, Shawver J, Kenmuir CL, Tietjen GE, Korsnack A et al (2018) Mechanical thrombectomy in elderly stroke patients with mild to-moderate baseline disability. Intervent Neurol 7:246-255

55. Salwi S, Cutting S, Salgado AD, Espaillat K, Fusco MR, Froehler MT et al (2020) Mechanical thrombectomy in patients with ischemic stroke with prestroke disability. Stroke 51:1539-1545

56. Jahan R, Saver JL, Schwamm LH, Fonarow GC, Liang L, Matsouaka RA, et al. Association between time to treatment with endovascular reperfusion therapy and outcomes in patients with acute ischemic stroke treated in clinical practice. JAMA — Journal of the American Medical Association. 2019;322:252-263

57. Hacke W, Kaste M, Bluhmki E, Brozman M, Dávalos A, Guidetti D et al (2008) Thrombolysis with alteplase 3 to 4.5 hours after acute ischemic stroke. N Engl J Med 359:1317-1329

58. Thomalla G, Simonsen CZ, Boutitie F, Andersen G, Berthezene $Y$, Cheng B et al (2018) Mri-guided thrombolysis for stroke with unknown time of onset. N Engl J Med 379:611-622

59. Coutinho JM, Liebeskind DS, Slater L-A, Nogueira RG, Clark W, Dávalos A et al (2017) Combined intravenous thrombolysis and thrombectomy vs thrombectomy alone for acute ischemic stroke: $A$ pooled analysis of the swift and star studies. jama Neurol 74:268-274

60. Goyal N, Tsivgoulis G, Frei D, Turk A, Baxter B, Froehler MT et al (2018) Comparative safety and efficacy of combined ivt and $\mathrm{mt}$ with direct $\mathrm{mt}$ in large vessel occlusion. Neurology 90:e1274

61. Menon BK, Al-Ajlan FS, Najm M, Puig J, Castellanos M, Dowlatshahi $D$, et al. Association of clinical, imaging, and thrombus characteristics with recanalization of visible intracranial occlusion in patients with acute ischemic stroke. JAMA-Journal of the American Medical Association. 2018;320:1017-1026

62. Alves Heitor C, Treurniet KM, Jansen IGH, Yoo AJ, Dutra BG, Zhang Getal (2019) Thrombus migration paradox in patients with acute ischemic stroke. Stroke 50:3156-3163

63. Flint Alexander C, Avins AL, Eaton A, Uong $S$, Cullen SP, Hsu DP et al (2020) Risk of distal embolization from tpa (tissue-type plasminogen activator) administration prior to endovascular stroke treatment. Stroke 51:2697-2704

64. Suzuki K, Matsumaru Y, Takeuchi M, Morimoto M, Kanazawa R, Takayama Y, et al. Effect of mechanical thrombectomy without vs with intravenous thrombolysis on functional outcome among patients with acute ischemic stroke: The skip randomized clinical trial. JAMA. 2021;325:244-253

65. Yang $P$, Zhang Y, Zhang L, Zhang Y, Treurniet KM, Chen W et al (2020) Endovascular thrombectomy with or without intravenous alteplase in acute stroke. NEngl J Med 382:1981-1993

66. Zi W, Qiu Z, Li F, Sang H, Wu D, Luo W et al (2021) Effect of endovascular treatment alone vs intravenous alteplase plus endovascular treatment on functional independence in patients with acute ischemic stroke: The devt randomized clinical trial. JAMA 325:234-243

67. Tsai CF, Thomas B, Sudlow CL (2013) Epidemiology of stroke and its subtypes in chinese vs white populations: A systematic review. Neurology 81:264-272

68. Campbell BCV, Mitchell PJ, Churilov L, Yassi N, Kleinig TJ, Dowling RJ etal (2018) Tenecteplaseversus alteplase before thrombectomy for ischemic stroke. N Engl J Med 378:1573-1582 


\section{Leitthema}

69. Saver JL, Goyal M, Van Der Lugt A, Menon BK Majoie CBLM, Dippel DW et al (2016) Time to treatment with endovascular thrombectomy and outcomes from ischemic stroke: Ameta-analysis. jama-journal Am Med Assoc 316:1279-1288

70. Schlemm L, Endres M, Nolte CH (2020) Bypassing the closest stroke center for thrombectomy candidates what additional delay to thrombolysis is acceptable? Stroke, S867-875

71. Duvekot MHC, Venema E, Rozeman AD, Moudrous W, Vermeij FH, Biekart M et al (2021) Comparison of eight prehospital stroke scales to detect intracranial large-vessel occlusion in suspected stroke (presto): A prospective observational study. Lancet Neurol. https://doi.org/10.1016/S14744422(20)30439-7

72. Nguyen TTM, van den Wijngaard IR, Bosch J, van Belle E, van Zwet EW, Dofferhoff-Vermeulen T et al (2020) Comparison of prehospital scales for predicting large anterior vessel occlusion in the ambulance setting. JAMA Neurol. https://doi.org/ 10.1161/STROKEAHA.120.033775

73. Mazya MV, Berglund A, Ahmed N, Von Euler M, Holmin S, Laska AC et al (2020) Implementation of a prehospital stroke triage system using symptom severity and teleconsultation in the stockholm stroke triage study. JAMA Neurol. https://doi.org/ 10.1001/jamaneurol.2020.0319

74. Fatima N, Saqqur M, Hussain MS, Shuaib A (2020) Mobile stroke unit versus standard medical care in the management of patients with acute stroke: $A$ systematic review and meta-analysis. Int J Stroke 15:595-608

75. Psychogios MN, Behme D, Schregel K, Tsogkas I, Maier IL, Leyhe JR, et al. One-stop management of acute stroke patients minimizing door-toreperfusion times. Stroke. 2017;48:3152-3155

76. Ospel JM, Almekhlafi MA, Menon BK, Kashani N, Chapot R, Fiehler J et al (2020) Workflow patterns and potential for optimization in endovascular stroke treatment across the world: Results from a multinational survey. J Neurolntervent Surg. https://doi.org/10.1136/neurintsurg-2020015902

77. Psychogios MN, Maier IL, Tsogkas I, Hesse AC, Brehm A, Behme D et al (2019) Onestop management of 230 consecutive acute stroke patients: Report of procedural times and clinical outcome. JCM. https://doi.org/10.3390/ jcm8122185

78. Tung Eric L, McTaggart RA, Baird Grayson L Yaghi S, Hemendinger M, Dibiasio EL et al (2017) Rethinking thrombolysis in cerebral infarction $2 \mathrm{~b}$. Stroke 48:2488-2493

79. Goyal N, Tsivgoulis G, Frei D, Turk A, Baxter B, Froehler MT et al (2019) Comparative safety and efficacy of modified tici $2 b$ and tici 3 reperfusion in acute ischemic strokes treated with mechanical thrombectomy. Neurosurgery $84: 680-686$

80. Maus V, Henkel S, Riabikin A, Riedel C, Behme D, Tsogkas I et al (2019) The save technique: Largescale experience for treatment of intracranial large vessel occlusions. clinical Neuroradiol 29:669-676

81. Kaesmacher J, Maegerlein C, Zibold F, Wunderlich S, Zimmer C, Friedrich B (2018) Improving mtici2b reperfusion to $\mathrm{mtici} 2 \mathrm{c} / 3$ reperfusions: A retrospective observational study assessing technical feasibility, safety and clinical efficacy. Eur Radiol 28:274-282

82. Behme D, Gera RG, Tsogkas I, Colla R, Liman J, Maier ILetal (2020) Impact of time on thrombolysis in cerebral infarction score results. Clin Neuroradiol 30:345-353

83. Kaesmacher J, Ospel JM, Meinel TR, Boulouis G, Goyal M, Campbell BCV, et al. Thrombolysis in cerebral infarction $2 \mathrm{~b}$ reperfusions: To treat or to stop? Stroke. 2020;51:3461-3471

84. Ferriero Donna M, Fullerton HJ, Timothy BJ, Billinghurst L, Daniels SR, DeBaun MR et al (2019) Management of stroke in neonates and children: A scientific statement from the american heart association/american stroke association. Stroke 50:e51-e96

Hier steht eine Anzeige. 